ITP-UH-14-95

April 1995

hep-th/9504089

\title{
The Supereigenvalue Model in the Double-Scaling Limit
}

\author{
Jan C. Plefka \\ Institut für Theoretische Physik, Universität Hannover \\ Appelstraße 2, 30167 Hannover, Germany \\ plefka@itp.uni-hannover.de
}

\begin{abstract}
The double-scaling limit of the supereigenvalue model is performed in the moment description. This description proves extremely useful for the identification of the multi-critical points in the space of bosonic and fermionic coupling constants. An iterative procedure for the calculation of higher-genus contributions to the free energy and to the multi-loop correlators in the double-scaling limit is developed. We present the general structure of these quantities at genus $g$ and give explicit results up to and including genus two.
\end{abstract}

\footnotetext{
†Supported by the 'Studienstiftung des Deutschen Volkes'
} 


\section{Introduction}

To date the most promising discrete approach to 2D supergravity coupled to minimal superconformal models is supplied by the supereigenvalue model proposed by Alvarez-Gaumé et al. [1]. It is formulated in terms of a collection of $N$ Grassmann even and odd variables, the "supereigenvalues", as well as a set of even and odd coupling constants. For a detailed review see ref. [2]. There still is no progress towards an understanding of the model on the basis of a generalized matrix model, which might provide us with a geometrical picture in the form of discretized superRiemann surfaces. I Nevertheless many of the well known features of the hermitian matrix model, such as the genus expansion, the Virasoro constraints, the loop equations, the moment description and the loop insertion operators [4, find their supersymmetric counterparts in the supereigenvalue model. From this point of view the supereigenvalue model appears as the natural supersymmetric generalization of the hermitian one-matrix model.

The supereigenvalue model is solvable nonperturbatively in coupling constants but perturbatively in its genus expansion. The solution is based on a set of superloop equations obeyed by the superloop correlators. Away from the double scaling limit these equations were first solved for general potentials in the planar limit in ref. [5]. In the moment description the computation of higher genus contributions could be automatized by an iterative procedure yielding results for general potentials and in principle arbitrary genus [6], representing a generalization of this very effective method for the hermitian matrix model [4]. Explicit results were given for genus one. An alternative approach was pursued by the authors of refs. [7, 8] who managed to directly integrate out the Grassmann-odd variables on the level of the partition function, uncovering the maximally quadratic dependence of the free energy on the fermionic coupling constants. The supereigenvalue model also displays a connection to supersymmetric integrable models [9].

In order to make contact with continuum physics the supereigenvalue model has to be studied in its double-scaling limit. This was done for symmetric bosonic potentials in ref. [1] in the planar limit, the case of general potentials was solved in ref. [5] for genus zero, one and partially for genus two. By making use of these results the authors of ref. [10] developed a precise dictionary between continuum $N=1$ super-Liouville amplitudes and supereigenvalue correlators.

In this paper the methods of the iterative solution of the supereigenvalue model [6] are applied to the double-scaling limit. The moment description turns out to be extremely useful for the determination of the critical points in the space of coupling constants. Just as in the hermitian matrix model Kazakov multicritical points [11 appear, related to extra zeros of the eigenvalue densities accumulating at one endpoint of the support. We identify the scaling properties of the moments and basis functions, confirming the results of refs. [1, 5]. The iterative procedure may be optimized to only produce terms relevant in the double-scaling limit. With these methods at hand we are able to state the general structure of the double scaled

\footnotetext{
${ }^{1}$ The only attempt in this direction is ref. [3]
} 
superloop correlators and the free energy. The iteration is perfectly suited for implementation on a computer algebra system. Explicit results are stated up to and including genus two.

The paper is organized as follows: In section 2 we briefly review the supereigenvalue model, the superloop equations and their iterative solution. In section 3 the double-scaling limit is performed. The multi-critical points in the moment description are identified and the scaling behaviour of moments and basis functions is obtained. We proceed to develop the iterative process in the scaling limit, state the general structure of the superloop correlators and the free energy and present our explicit results. Section 4 finally contains the conclusions and a short discussion of future perspectives.

\section{Iterative Solution of the Supereigenvalue Model}

In the following we give a brief account of the iterative procedure to solve the supereigenvalue model genus by genus for general potentials. A more elaborate description may be found in ref. [6]. The aim of this paper is to apply these methods to the double-scaling limit.

\subsection{Superloop Insertion Operators}

The supereigenvalue model [1] is defined by the partition function

$$
\mathcal{Z}=e^{N^{2} F}=\int\left(\prod_{i=1}^{N} d \lambda_{i} d \theta_{i}\right) \prod_{i<j}\left(\lambda_{i}-\lambda_{j}-\theta_{i} \theta_{j}\right) \exp \left(-N \sum_{i=1}^{N}\left[V\left(\lambda_{i}\right)-\theta_{i} \Psi\left(\lambda_{i}\right)\right]\right),
$$

where the integration is over a set of $N$ bosonic and fermionic variables, denoted by $\lambda_{i}$ and $\theta_{i}$ respectively. $N$ is even. Moreover the Grassmann even and odd potentials take the general form

$$
V\left(\lambda_{i}\right)=\sum_{k=0}^{\infty} g_{k} \lambda_{i}^{k} \quad \text { and } \quad \Psi\left(\lambda_{i}\right)=\sum_{k=0}^{\infty} \xi_{k+1 / 2} \lambda_{i}{ }^{k}
$$

the $g_{k}$ and and $\xi_{k+1 / 2}$ being Grassmann even and odd coupling constants, respectively. The basic observables of this model are the connected $(n \mid m)$-superloop correlators

$$
\begin{aligned}
& W\left(p_{1}, \ldots, p_{n} \mid q_{1}, \ldots, q_{m}\right)= \\
& \quad N^{n+m-2}\left\langle\sum_{i_{1}} \frac{\theta_{i_{1}}}{p_{1}-\lambda_{i_{1}}} \cdots \sum_{i_{n}} \frac{\theta_{i_{n}}}{p_{n}-\lambda_{i_{n}}} \sum_{j_{1}} \frac{1}{q_{1}-\lambda_{j_{1}}} \cdots \sum_{j_{m}} \frac{1}{q_{m}-\lambda_{j_{m}}}\right\rangle_{\mathrm{conn}},
\end{aligned}
$$

where the expectation value is defined in the conventional way and where "conn" refers to the connected part. These superloop correlators act as generating functionals for higher-point correlators like $\left\langle\sum_{i} \theta_{i} \lambda_{i}{ }^{k} \sum_{j} \lambda_{j}{ }^{l}\right\rangle$ upon expansion in $p_{i}$ and 
$q_{i}$. The connected $(n \mid m)$-superloop correlators are related to the free energy by the application of the superloop insertion operators $\delta / \delta V(p)$ and $\delta / \delta \Psi(p)$ :

$$
W\left(p_{1}, \ldots, p_{n} \mid q_{1}, \ldots, q_{m}\right)=\frac{\delta}{\delta \Psi\left(p_{1}\right)} \ldots \frac{\delta}{\delta \Psi\left(p_{n}\right)} \frac{\delta}{\delta V\left(q_{1}\right)} \cdots \frac{\delta}{\delta V\left(q_{m}\right)} F
$$

where

$$
\frac{\delta}{\delta V(p)}=-\sum_{k=0}^{\infty} \frac{1}{p^{k+1}} \frac{\partial}{\partial g_{k}} \quad \text { and } \quad \frac{\delta}{\delta \Psi(p)}=-\sum_{k=0}^{\infty} \frac{1}{p^{k+1}} \frac{\partial}{\partial \xi_{k+1 / 2}} .
$$

Hence from the one-superloop correlators $W(\mid p)$ and $W(p \mid)$ (or the free energy $F$ ) all the multi-superloop correlators can be obtained by application of the superloop insertion operators.

With the normalizations chosen above, the genus expansion of the correlators reads

$$
W\left(p_{1}, \ldots, p_{n} \mid q_{1}, \ldots, q_{m}\right)=\sum_{g=0}^{\infty} \frac{1}{N^{2 g}} W_{g}\left(p_{1}, \ldots, p_{n} \mid q_{1}, \ldots, q_{m}\right) .
$$

Similarly we have

$$
F=\sum_{g=0}^{\infty} \frac{1}{N^{2 g}} F_{g}
$$

for the free energy.

\subsection{Superloop Equations}

The genus $g$ contribution to the one-superloop correlators $W(\mid p)$ and $W(p \mid)$ may be found by solving the superloop equations of the model by iteration. These two equations express the invariance of the partition function (2.1) under field redefinitions as shown in ref. [6].

The Grassmann-odd superloop equation takes the form

$$
\oint_{C} \frac{d \omega}{2 \pi i} \frac{V^{\prime}(\omega)}{p-\omega} W(\omega \mid)+\oint_{C} \frac{d \omega}{2 \pi i} \frac{\Psi(\omega)}{p-\omega} W(\mid \omega)=W(p \mid) W(\mid p)+\frac{1}{N^{2}} W(p \mid p)
$$

and its counterpart, the Grassmann-even superloop equation, reads

$$
\begin{gathered}
\oint_{C} \frac{d \omega}{2 \pi i} \frac{V^{\prime}(\omega)}{p-\omega} W(\mid \omega)+\oint_{C} \frac{d \omega}{2 \pi i} \frac{\Psi^{\prime}(\omega)}{p-\omega} W(\omega \mid)-\frac{1}{2} \frac{d}{d p} \oint_{C} \frac{d \omega}{2 \pi i} \frac{\Psi(\omega)}{p-\omega} W(\omega \mid)= \\
\frac{1}{2}\left[W(\mid p)^{2}-W(p \mid) W^{\prime}(p \mid)+\frac{1}{N^{2}}\left(W(\mid p, p)-\left.\frac{d}{d q} W(p, q \mid)\right|_{p=q}\right)\right] .
\end{gathered}
$$


In the derivation we have assumed a one-cut structure of the loop correlators, i.e. in the limit $N \rightarrow \infty$ we assume that the eigenvalues are contained in a finite interval $[x, y] . C$ is a curve around the cut. Moreover eqs. (2.8) and (2.9) encode the superVirasoro constraints $G_{k+1 / 2} \mathcal{Z}=0$ and $L_{k} \mathcal{Z}=0$ for $k \geq-1$, which the model obeys by construction [四]. As demonstrated in ref. [6] these equations are accessible to an iterative solution in genus $g$. The strategy consist in employing the observation of refs. [7, 8] that the free energy $F$ depends at most quadratically on fermionic coupling constants. By eq. (2.4) this allows us to write

$$
W(p \mid)=v(p) \quad \text { and } \quad W(\mid p)=u(p)+\widehat{u}(p) .
$$

Here $v(p)$ is of order one, $u(p)$ of order zero and $\widehat{u}(p)$ of order two in the fermionic coupling constants $\xi_{k+1 / 2}$. Plugging these relations and the genus expansion (2.6) into the superloop equations (2.8) and (2.9) yields a set of four equations at each genus sorted by their order in fermionic coupling constants for the quantities $v_{g}(p)$, $u_{g}(p)$ and $\widehat{u}_{g}(p)$.

The equation of order 0 in fermionic couplings at genus $g \geq 1$ reads

$$
\begin{aligned}
\left(\widehat{V}^{\prime}-u_{0}(p)\right) u_{g}(p)= & \frac{1}{2} \sum_{g^{\prime}=1}^{g-1} u_{g^{\prime}}(p) u_{g-g^{\prime}}(p)+\frac{1}{2} \frac{\delta}{\delta V(p)} u_{g-1}(p) \\
& -\left.\frac{1}{2} \frac{d}{d q} \frac{\delta}{\delta \Psi(p)} v_{g-1}(q)\right|_{p=q}
\end{aligned}
$$

where we have introduced the linear operator $\widehat{V}^{\prime}$ defined by

$$
\widehat{V}^{\prime} f(p)=\oint_{C} \frac{d \omega}{2 \pi i} \frac{V^{\prime}(\omega)}{p-\omega} f(\omega) .
$$

The equation of order 1 at $g \geq 1$ takes the form

$$
\begin{aligned}
\left(\widehat{V}^{\prime}-u_{0}(p)\right) v_{g}(p)= & -\left(\widehat{\Psi}-v_{0}(p)\right) u_{g}(p)+\sum_{g^{\prime}=1}^{g-1} v_{g^{\prime}}(p) u_{g-g^{\prime}}(p) \\
& +\frac{\delta}{\delta V(p)} v_{g-1}(p)
\end{aligned}
$$

analogously the operator $\widehat{\Psi}$ is defined by

$$
\widehat{\Psi} f(p)=\oint_{C} \frac{d \omega}{2 \pi i} \frac{\Psi(\omega)}{p-\omega} f(\omega) .
$$

In fact for $g \geq 1$ there is no need to solve the equations of order 2 and 3 , as the knowledge of the quantities $u_{g}(p)$ and $v_{g}(p)$ (up to a zero mode) suffices to compute the free energy $F_{g}$ and the remaining $\widehat{u}_{g}[6]$. It is the remarkable structure of eqs. (2.11) and (2.13) which allows us to develop an iterative procedure to solve for $u_{g}$ and $v_{g}$. At genus $g$ the right hand side of (2.11) only contains contributions from 
lower genera, knowing these the inversion of the operator $\left(\widehat{V}^{\prime}-u_{0}(p)\right)$ then yields $u_{g}(p)$. Similarly the right hand side of eq. (2.13) contains $u_{g}$ and terms of lower genera, allowing the computation of $v_{g}(p)$ (up to the zero mode of the operator $\left.\left(\widehat{V}^{\prime}-u_{0}(p)\right)\right)$. Hence the seed of the solution is $u_{0}$ and $v_{0}$. Let us now turn to an important tool of the solution, the change of variables from coupling constants to moments and the introduction of basis functions.

\subsection{Moments and Basis Functions}

The planar as well as the higher genera contributions to the loop correlators and the free energy are most easily expressed by introducing instead of the couplings $\left\{g_{k}, \xi_{k+1 / 2}\right\}$ a set of Grassmann even and odd moments $\left\{M_{k}, J_{k}, \Xi_{k}, \Lambda_{k}\right\}$. We replace the bosonic couplings $g_{k}$ by the moments [- []

$$
\begin{aligned}
M_{k} & =\oint_{C} \frac{d \omega}{2 \pi i} \frac{V^{\prime}(\omega)}{(\omega-x)^{k}} \frac{1}{[(\omega-x)(\omega-y)]^{1 / 2}}, \quad k \geq 1 \\
J_{k} & =\oint_{C} \frac{d \omega}{2 \pi i} \frac{V^{\prime}(\omega)}{(\omega-y)^{k}} \frac{1}{[(\omega-x)(\omega-y)]^{1 / 2}}, \quad k \geq 1,
\end{aligned}
$$

and the fermionic couplings $\xi_{k+1 / 2}$ by

$$
\begin{aligned}
& \Xi_{k}=\oint_{C} \frac{d \omega}{2 \pi i} \frac{\Psi(\omega)}{(\omega-x)^{k}}[(\omega-x)(\omega-y)]^{1 / 2}, \quad k \geq 1 \\
& \Lambda_{k}=\oint_{C} \frac{d \omega}{2 \pi i} \frac{\Psi(\omega)}{(\omega-y)^{k}}[(\omega-x)(\omega-y)]^{1 / 2}, \quad k \geq 1 .
\end{aligned}
$$

One advantage of this change of variables is that the contributions at genus $g \geq 1$ to the loop correlators and the free energy depend only on a finite number of moments, e.g. $F_{g}$ depends at most on $2 \times 3 g$ bosonic and $2 \times(3 g+1)$ fermionic moments [6]. As opposed to this $F_{g}$ is a function of the entire set of coupling constants $\left\{g_{k}, \xi_{k+1 / 2}\right\}$. Furthermore the description in terms of moments will prove extremely useful in the double-scaling limit.

For the development of the iterative procedure we further introduce the basis functions $\chi^{(n)}(p)$ and $\Psi^{(n)}(p)$ recursively

$$
\begin{aligned}
\chi^{(n)}(p) & =\frac{1}{M_{1}}\left(\phi_{x}^{(n)}(p)-\sum_{k=1}^{n-1} \chi^{(k)}(p) M_{n-k+1}\right), \\
\Psi^{(n)}(p) & =\frac{1}{J_{1}}\left(\phi_{y}^{(n)}(p)-\sum_{k=1}^{n-1} \Psi^{(k)}(p) J_{n-k+1}\right),
\end{aligned}
$$

where 


$$
\phi_{x}^{(n)}(p)=(p-x)^{-n-1 / 2}(p-y)^{-1 / 2}, \quad \phi_{y}^{(n)}(p)=(p-x)^{-1 / 2}(p-y)^{-n-1 / 2},
$$

following ref. 四. $\chi^{(n)}(p)$ and $\Psi^{(n)}(p)$ are basis functions of the linear operator $\widehat{V}^{\prime}$ appearing in the superloop equations in the sense that

$$
\begin{array}{ll}
\left(\widehat{V^{\prime}}-u_{0}(p)\right) \chi^{(n)}(p)=\frac{1}{(p-x)^{n}}, \quad n \geq 1, \\
\left(\widehat{V^{\prime}}-u_{0}(p)\right) \Psi^{(n)}(p)=\frac{1}{(p-y)^{n}}, \quad n \geq 1 .
\end{array}
$$

Note that $\phi_{x}^{(0)}(p)=\phi_{y}^{(0)}(p) \equiv \phi^{(0)}(p)$ lies in the kernel of $\left(\widehat{V}^{\prime}-u_{0}(p)\right)$ and constitutes the zero mode of $v_{g}(p)$ mentioned above.

\subsection{The Planar Solution}

With these definitions at hand we may now state the planar $(g=0)$ solution of eqs. (2.8) and (2.9) obtained in refs. [5, 6]:

$$
\begin{aligned}
W_{0}(\mid p)= & \oint_{C} \frac{d \omega}{2 \pi i} \frac{V^{\prime}(\omega)}{p-\omega}\left[\frac{(p-x)(p-y)}{(\omega-x)(\omega-y)}\right]^{1 / 2} \\
& +\frac{1}{4} \frac{\Xi_{2}\left(\Xi_{1}-\Lambda_{1}\right)}{M_{1}(x-y)} \phi_{x}^{(1)}(p)+\frac{1}{4} \frac{\Lambda_{2}\left(\Xi_{1}-\Lambda_{1}\right)}{J_{1}(x-y)} \phi_{y}^{(1)}(p) \\
W_{0}(p \mid)= & \oint_{C} \frac{d \omega}{2 \pi i} \frac{\Psi(\omega)}{p-\omega}\left[\frac{(\omega-x)(\omega-y)}{(p-x)(p-y)}\right]^{1 / 2}+\frac{1}{2} \frac{\Xi_{1}+\Lambda_{1}}{[(p-x)(p-y)]^{1 / 2}} .
\end{aligned}
$$

Here the endpoints $x$ and $y$ of the cut on the real axis are determined by the boundary conditions

$$
0=\oint_{C} \frac{d \omega}{2 \pi i} \frac{V^{\prime}(\omega)}{\sqrt{(\omega-x)(\omega-y)}}, \quad 1=\oint_{C} \frac{d \omega}{2 \pi i} \frac{\omega V^{\prime}(\omega)}{\sqrt{(\omega-x)(\omega-y)}}
$$

deduced from our knowledge that $W(\mid p)=1 / p+\mathcal{O}\left(p^{-2}\right)$. This is also the reason why there are no zero mode contributions possible for the $W_{g}(\mid p)$. We shall make use of the following rewriting of the purely bosonic part of $W(\mid p)$ 12

$$
u_{0}(p)=V^{\prime}(p)-\frac{1}{2}[(p-x)(p-y)]^{1 / 2} \sum_{q=1}^{\infty}\left\{(p-x)^{q-1} M_{q}+(p-y)^{q-1} J_{q}\right\},
$$


derived by deforming the contour integral in eq. (2.24) into one surrounding the point $p$ and the other encircling infintity. To take the residue at infinity one rewrites $(p-\omega)^{-1}$ as

$$
\frac{1}{p-\omega}=\frac{1}{2} \frac{1}{(p-x)-(\omega-x)}+\frac{1}{2} \frac{1}{(p-y)-(\omega-y)}
$$

and expands in $\left(\frac{p-x}{\omega-x}\right)$ and $\left(\frac{p-y}{\omega-y}\right)$ respectively. Doing this for the fermionic $W_{0}(p \mid)$ yields

$$
v_{0}(p)=\Psi(p)-\frac{1}{2}[(p-x)(p-y)]^{-1 / 2} \sum_{q=2}^{\infty}\left\{(p-x)^{q-1} \Xi_{q}+(p-y)^{q-1} \Lambda_{q}\right\} .
$$

It is important to realize that the bracketed terms in eq. (2.27) as well as in eq. (2.29) are actually identical. Here we see that the planar solution is special in the sense that it depends on the full set of moments. Interestingly enough this is not the case for higher genera.

\section{The Double-Scaling Limit}

Similar to the situation in the hermitian matrix model the "naive" $N \rightarrow \infty$ continuum limit of the supereigenvalue model is unsatisfactory as it leaves us only with the planar contributions, easily seen from eqs. (2.6) and (2.7). More interesting from the point of view of continuum physics is the double-scaling limit. Crucial for this scenario is the observation that there exists a subspace in the space of couplings $\left\{g_{k}, \xi_{k+1 / 2}\right\}$ where all higher genus contributions to the free energy $F_{g}$ diverge. This enables us to take the double-scaling limit, where one simultaneously approaches the critical subspace of couplings and takes $N \rightarrow \infty$, giving contributions to the free energy from all genera. Let us see how this works in detail.

\subsection{Scaling of Moments and Basis Functions}

The analysis of the scaling behaviour for the bosonic quantities was carried out by Ambjørn et al. [4, 12] in the framework of the hermitian matrix model. Consider the case of generic, i.e. non-symmetric, potentials $V(p)$ and $\Psi(p)$. The $m$ 'th multicritical point is reached when the eigenvalue density $\left(u_{0}(p)-V^{\prime}(p)\right)$ of eq. (2.27), which under normal circumstances vanishes as a square root on both ends of its support, aquires $(m-1)$ additional zeros at one end of the cut, say $x$. The condition for being at an $m$ 'th multicritical point then simply is

$$
M_{1}^{c}=M_{2}^{c}=\cdots M_{m-1}^{c}=0, \quad M_{k}^{c} \neq 0, \quad k \geq m, \quad J_{l}^{c} \neq 0, \quad l \geq 1,
$$

defining a critical subspace in the space of bosonic couplings $g_{k}$. Denote by $g_{k}^{c}$ a particular point in this subspace for which the eigenvalue distribution is confined to 
the interval $\left[x_{c}, y_{c}\right]$. If we now move away from this point the conditions of eq. (3.1) will no longer be fulfilled and the cut will move to the intervall $[x, y]$. Assume we control this movement by the parameter $a$ and set [1]

$$
\begin{aligned}
x & =x_{c}-a \Lambda^{1 / m} \\
p & =x_{c}+a \pi
\end{aligned}
$$

The scaling of $p$ and the introduction of its scaling variable $\pi$ is necessary in order to speak of the double-scaling limit of the superloop correlators. $\Lambda$ plays the role of the cosmological constant. We will now deduce the scaling of $y$ by further assuming that the critical subspace $\left\{g_{k}^{c}\right\}$ is reached as

$$
g_{k}=g \cdot g_{k}^{c}
$$

where $g$ is a function of $a$ to be determined. Imposing the boundary conditions (2.26) yields

$$
y-y_{c} \sim a^{m}, \text { and } g-1 \sim a^{m} .
$$

Knowing this one easily computes the $m$ 'th multicritical scaling behaviour of the bosonic moments

$$
M_{k} \sim a^{m-k}, \quad k \in[1, m-1],
$$

while the higher $M$-moments and the $J$-moments do not scale.

Moreover the functions $\phi_{x}^{(n)}(p)$ and $\phi_{y}^{(n)}(p)$ are found to behave like

$$
\phi_{x}^{(n)}(p) \sim a^{-n-1 / 2}, \quad \phi_{y}^{(n)}(p) \sim a^{-1 / 2},
$$

from which one proves the scaling behaviour of the basis functions

$$
\chi^{(n)}(p) \sim a^{-m-n+1 / 2}, \quad \Psi^{(n)}(p) \sim a^{-1 / 2},
$$

following ref. 4 .

Let us now turn to the scaling of the fermionic moments $\Xi_{k}$ and $\Lambda_{k}$. Similar to the bosonic case the function $\left(v_{0}(p)-\Psi(p)\right)$ of eq. (2.29) usually vanishes at the endpoints of the cut like a square root. We will fine tune the coupling constants $\xi_{k+1 / 2}$ in such a manner that $(n-1)$ extra zeros accumulate at $x$, i.e.

$$
\Xi_{2}^{c}=\cdots \Xi_{n-1}^{c}=0, \quad \Xi_{k}^{c} \neq 0, \quad k \geq n, \quad \Lambda_{l} \neq 0, \quad l \geq 2,
$$

where $\Xi_{k}^{c} \equiv \Xi_{k}\left[x_{c}, y_{c}, \xi_{k+1 / 2}^{c}\right]$. In addition the results of ref. [6] tell us that the moments $\Xi_{1}$ and $\Lambda_{1}$ will always appear in the combination $\left(\Xi_{1}-\Lambda_{1}\right)$. This suggests to impose the constraint $\Xi_{1}^{c}-\Lambda_{1}^{c}=0$ on these moments. As there is no analogue to the boundary conditions (2.26) for the fermionic quantities we are free to choose the scaling of the coupling constants $\xi_{k+1 / 2}$. We set 


$$
\xi_{k+1 / 2}=a^{1 / 2} \xi_{k+1 / 2}^{c}
$$

and will comment on this choice later on. From this one derives $\left(\Xi_{1}-\Lambda_{1}\right) \sim a^{n-1 / 2}$ and $\Xi_{k} \sim a^{n-k+1 / 2}$ for $k \in[2, n-1]$. All other fermionic moments scale uniformly with $a^{1 / 2}$. So far the fermionic scaling is completely independent of the bosonic scaling, governed by the integer $n$. We shall, however, introduce the requirement that the scaling part of the bosonic one-superloop correlator of eq. (2.24) scales uniformly, i.e. we require $\left(u_{0}(p)-V^{\prime}(p)\right)$ and $\widehat{u}_{0}(p)$ to scale in the same way [5]. As $\left(u_{0}(p)-V^{\prime}(p)\right) \sim a^{m-1 / 2}$ we arrive at the following condition on $n$ :

$$
n=m+1
$$

And therefore

$$
\begin{array}{ll}
\Xi_{1}-\Lambda_{1} \sim a^{m+1 / 2}, & \Xi_{k} \sim a^{m-k+3 / 2}, \quad k \in[2, m] \\
\Xi_{k} \sim a^{1 / 2}, \quad k>m & \Lambda_{l} \sim a^{1 / 2}, \quad l>1 .
\end{array}
$$

The double-scaling limit is now defined by letting $N \rightarrow \infty$ and $a \rightarrow 0$ but keeping the string coupling constant $\alpha=a^{-2 m-1} N^{-2}$ fixed. ?

The above scaling behaviour may be shown to be equivalent to the one obtained in ref. [5]. Based on these results Abdalla and Zadra [10] proved that the central charge of the superconformal field theory described in the continuum is given by

$$
\widehat{c}=1-\frac{(2 m-1)^{2}}{m}=0,-\frac{7}{2}, \ldots \quad m \geq 1 .
$$

Similar to the situation in the bosonic model the $m=1$ fixed point may not be reached by the described techniques and should be treated seperately.

\subsection{The Iteration for $u_{g}(p)$ and $v_{g}(p)$}

By making use of the above scaling properties of the moments and basis functions we may now develop the iterative procedure which allows us to calculate directly the double-scaling relevant versions of $u_{g}(p)$ and $v_{g}(p)$. The iterative scheme described in ref. [6] is quite simple: By eqs. (2.22) and (2.23) every $u_{g}$ and $v_{g}$ may be written as a linear combination of basis functions $\chi^{(n)}$ and $\Psi^{(n)}$, where the coefficients of this expansion are simply read off the poles at $x$ and $y$ of the right hand sides of

\footnotetext{
${ }^{2}$ Let us now comment on the choice of eq. (3.10). At first sight one might have expected a scaling like $\xi_{k+1 / 2}=[1+o(a)] \xi_{k+1 / 2}^{c}$. This turns out to be inconsistent because then the condition (3.11) demands $n$ to be half-integer which it can not be. If one takes the more general ansatz $\xi_{k+1 / 2}=a^{l} \xi_{k+1 / 2}^{c}$ the condition of uniform scaling of $W(\mid p)$ yields the allowed sequence $\{n, l\}=\{m+1,1 / 2\},\{m, 3 / 2\},\{m-1,5 / 2\}, \ldots$ The scaling of the lowest moments in eq. (3.12) remains unchanged, the uniform scaling however already starts with $\Xi_{n}$ scaling like $a^{l}$ and thus simply reduces the number of double-scaling relevant terms.
} 
the superloop equations (2.11) and (2.13) after a partial fraction decomposition. To optimize the procedure to only produce terms which are relevant in the doublescaling limit we have to analyze the operators appearing on the right hand sides of the superloop equations, i.e. the superloop insertion operators $\delta / \delta V(p)$ and $\delta / \delta \Psi(p)$ as well as $\left(\widehat{\Psi}-v_{0}(p)\right)$.

From the point of view of the $a \rightarrow 0$ limit the effect of a given operator in $\delta / \delta V(p)$ acting on an expression which scales with $a$ to some power is to lower this power by a certain amount. Carefully examining each term in $\delta / \delta V(p)$ shows that a is maximally lowered by a power of $(m+3 / 2)$. All operators which do not lower $a$ by this amount are subdominant in the scaling limit and may be neglected. The outcome of this analysis for $\delta / \delta V(p)$ is

$$
\begin{aligned}
\frac{\delta}{\delta V(p)_{x}}=\sum_{k=1}^{\infty} & \frac{\delta M_{k}}{\delta V(p)_{x}} \frac{\delta}{\delta M_{k}}+\frac{\delta x}{\delta V(p)} \frac{\delta}{\delta x} \\
& +\frac{\delta\left(\Xi_{1}-\Lambda_{1}\right)}{\delta V(p)_{x}} \frac{\delta}{\delta\left(\Xi_{1}-\Lambda_{1}\right)}+\sum_{k=2}^{\infty} \frac{\delta \Xi_{k}}{\delta V(p)_{x}} \frac{\delta}{\delta \Xi_{k}}
\end{aligned}
$$

where 3

$$
\begin{aligned}
\frac{\delta M_{k}}{\delta V(p)_{x}}=-\left(k+\frac{1}{2}\right) \phi_{x}^{(k+1)}(p)+\left(k+\frac{1}{2}\right) \frac{M_{k+1}}{M_{1}} \phi_{x}^{(1)}(p) & \frac{\delta x}{\delta V(p)}=\frac{1}{M_{1}} \phi_{x}^{(1)}(p) \\
\frac{\delta\left(\Xi_{1}-\Lambda_{1}\right)}{\delta V(p)_{x}} & =\frac{1}{2} \frac{\Xi_{2}}{M_{1}} \phi_{x}^{(1)}(p) \\
\frac{\delta \Xi_{k}}{\delta V(p)_{x}} & =\left(k-\frac{1}{2}\right) \frac{\Xi_{k+1}}{M_{1}} \phi_{x}^{(1)}(p)
\end{aligned}
$$

and

$$
\phi_{x}^{(n)}(p)=(p-x)^{-n-1 / 2} d_{c}^{-1 / 2}
$$

with $d_{c}=x_{c}-y_{c}$. Repeating this analysis for the fermionic superloop insertion operator $\delta / \delta \Psi(p)$ shows that here $a$ is maximally lowered by a power of $(m+1)$ and the relevant contributions are

$$
\frac{\delta}{\delta \Psi(p)_{x}}=\frac{\delta\left(\Xi_{1}-\Lambda_{1}\right)}{\delta \Psi(p)_{x}} \frac{\delta}{\delta\left(\Xi_{1}-\Lambda_{1}\right)}+\sum_{k=2}^{\infty} \frac{\delta \Xi_{k}}{\delta \Psi(p)_{x}} \frac{\delta}{\delta \Xi_{k}}
$$

with

\footnotetext{
${ }^{3}$ Here the subscript $x$ indicates that the critical behaviour is associated with the endpoint $x$
} 


$$
\begin{aligned}
\frac{\delta\left(\Xi_{1}-\Lambda_{1}\right)}{\delta \Psi(p)_{x}} & =-d_{c} \phi_{x}^{(0)}(p) \\
\frac{\delta \Xi_{k}}{\delta \Psi(p)_{x}} & =-d_{c} \phi_{x}^{(k-1)}(p) .
\end{aligned}
$$

Finally we state the double-scaling version of the operator $\left(\widehat{\Psi}-v_{0}(p)\right)$ acting on the function $\phi_{x}^{(n)}(p)$

$$
\left(\widehat{\Psi}-v_{0}(p)\right)_{x} \phi_{x}^{(n)}(p)=\sum_{k=1}^{n} \frac{\Xi_{n+2-k}}{d_{c}} \frac{1}{(p-x)^{k}}+\frac{\left(\Xi_{1}-\Lambda_{1}\right)}{2 d_{c}} \frac{1}{(p-x)^{n+1}} .
$$

Here the operator $\left(\widehat{\Psi}-v_{o}(p)\right)$ is seen to increase the power of $a$ of the expression it acts on by a factor of $m$.

We are now in a position to calculate the double-scaling limit of the right hand sides of the loop equations (2.11) and (2.13) for $u_{g}(p)$ and $v_{g}(p)$ provided we know the double scaled versions of $u_{1}(p), \ldots, u_{g-1}(p)$ and $v_{1}(p), \ldots, v_{g-1}(p)$. As all the $y$ dependence has disappeared we do not have to perform a decomposition of the result. Moreover no $J_{k}$ and $\Lambda_{k}$ dependent terms will contribute if we do not start out with any and we do not.

The starting point of the iterative procedure are of course the genus 0 correlators [6]. Keeping only the doublescaling relevant parts one has

$$
-\left.\frac{d}{d q} \frac{\delta v_{0}(q)}{\delta \Psi(p)_{x}}\right|_{p=q}=\frac{\delta u_{0}(p)}{\delta V(p)_{x}}=\frac{1}{8} \frac{1}{(p-x)^{2}}
$$

and

$$
\frac{\delta v_{0}(p)}{\delta V(p)_{x}}=\left[-\frac{\left(\Xi_{1}-\Lambda_{1}\right)}{4 d_{c} M_{1}}\right] \frac{1}{(p-x)^{3}}+\left[\frac{\Xi_{2}}{4 d_{c} M_{1}}\right] \frac{1}{(p-x)^{2}}
$$

The higher genus correlators $u_{g}(p)$ and $v_{g}(p)$ are expressed as linear combinations of the basis function $\chi^{(n)}(p)$ and take the general form [6]

$$
u_{g}(p)=\sum_{k=1}^{3 g-1} A_{g}^{(k)} \chi^{(k)}(p) \quad \text { and } \quad v_{g}(p)=\sum_{k=1}^{3 g} B_{g}^{(k)} \chi^{(k)}(p)+\kappa_{g} \phi^{(0)}(p)
$$

where $\kappa_{g}$ is the zero mode coefficient not determined by the first two superloop equations (2.11) and (2.13). Note that the $A_{g}^{(k)}$ coefficients should up to a factor of two be identical to those of the hermitian matrix model obtained in ref. [4]. We have calculated the $A_{g}^{(k)}$ and $B_{g}^{(k)}$ coefficients in the double-scaling limit for $g=1,2$ and 3 with the aid of Maple. The results of Ambjørn et al. [四] for the $A_{g}^{(k)}$ coefficients could be reproduced. 
Before we state our explicit results let us turn to the general scaling behaviour of the one-superloop correlators

$$
W_{g}(\mid p) \sim a^{(1-2 g)(m+1 / 2)-1}, \quad \text { and } \quad W_{g}(p \mid) \sim a^{(1-2 g)(m+1 / 2)-1 / 2},
$$

which one proves by induction. The structure of the coefficients $B_{g}^{(k)}$ is seen to be

$$
B_{g}^{(k)}=\sum_{\alpha_{j}, \beta}\left\langle\alpha_{1}, \ldots, \alpha_{s}, \beta \mid \alpha\right\rangle_{g, k} \frac{M_{\alpha_{1}} \ldots M_{\alpha_{s}} \Xi_{\beta}}{M_{1}{ }^{\alpha} d_{c}{ }^{g}},
$$

where the brackets denote rational numbers and where we write $\Xi_{1}$ for $\left(\Xi_{1}-\Lambda_{1}\right)$. One shows that the $\alpha, \alpha_{j}, \beta$ and $s$ obey the conditions

$$
\alpha=2 g+s-1, \quad \text { and } \quad \sum_{j=1}^{s}\left(\alpha_{j}-1\right)=3 g+1-\beta-k
$$

with $\alpha_{j} \in[2,3 g]$ and $\beta \in[1,3 g]$. For the zero mode coefficient $\kappa_{g}$ the general structure is given by the same expansion as eq. (3.28) with $k=0$. The conditions on $\alpha$, $\alpha_{j}, \beta$ and $s$ then read

$$
\alpha=2 g+s, \quad \text { and } \quad \sum_{j=1}^{s}\left(\alpha_{j}-1\right)=3 g+1-\beta
$$

where $\alpha_{j} \in[2,3 g]$ and $\beta \in[1,3 g+1]$. Similar relations hold for the $A_{g}^{(k)}$ [1].

The explicit results for the $B_{g}^{(k)}$ coefficients for $g=1$ and $g=2$ now read

$$
\begin{aligned}
B_{1}^{(1)}= & -\frac{\Xi_{3}}{8 M_{1} d_{c}}+\frac{M_{2} \Xi_{2}}{8 M_{1}{ }^{2} d_{c}}, \\
B_{1}^{(2)}= & \frac{M_{2}\left(\Xi_{1}-\Lambda_{1}\right)}{16 M_{1}{ }^{2} d_{c}}+\frac{\Xi_{2}}{8 M_{1} d_{c}}, \quad B_{1}^{(3)}=-\frac{5\left(\Xi_{1}-\Lambda_{1}\right)}{16 M_{1} d_{c}} . \\
B_{2}^{(1)}= & \frac{203 M_{2} \Xi_{5}}{128 d_{c}{ }^{2} M_{1}{ }^{4}}-\frac{145 M_{3}{ }^{2} \Xi_{2}}{128 M_{1}{ }^{5} d_{c}{ }^{2}}-\frac{105 \Xi_{6}}{128 d_{c}{ }^{2} M_{1}{ }^{3}}+\frac{63 M_{2}{ }^{3} \Xi_{3}}{32 M_{1}{ }^{6} d_{c}{ }^{2}} \\
& +\frac{105 M_{4} \Xi_{3}}{128 d_{c}{ }^{2} M_{1}{ }^{4}}+\frac{145 M_{3} \Xi_{4}}{128 d_{c}{ }^{2} M_{1}{ }^{4}}+\frac{105 M_{5} \Xi_{2}}{128 d_{c}{ }^{2} M_{1}{ }^{4}}-\frac{77 M_{4} M_{2} \Xi_{2}}{32 M_{1}{ }^{5} d_{c}{ }^{2}} \\
& -\frac{87 M_{3} M_{2} \Xi_{3}}{32 M_{1}{ }^{5} d_{c}{ }^{2}}+\frac{75 M_{3} M_{2}{ }^{2} \Xi_{2}}{16 M_{1}{ }^{6} d_{c}{ }^{2}}-\frac{63 M_{2}{ }^{4} \Xi_{2}}{32 M_{1}{ }^{7} d_{c}{ }^{2}}-\frac{63 M_{2}{ }^{2} \Xi_{4}}{32 M_{1}{ }^{5} d_{c}{ }^{2}}, \\
B_{2}^{(2)}= & -\frac{21 M_{2}{ }^{3} \Xi_{2}}{64 M_{1}{ }^{6} d_{c}{ }^{2}}-\frac{21 M_{2} \Xi_{4}}{64 d_{c}{ }^{2} M_{1}{ }^{4}}+\frac{77 M_{3} M_{2} \Xi_{2}}{128 M_{1}{ }^{5} d_{c}{ }^{2}}-\frac{35 M_{4} \Xi_{2}}{128 d_{c}{ }^{2} M_{1}{ }^{4}} \\
& +\frac{75 M_{3} M_{2}{ }^{2}\left(\Xi_{1}-\Lambda_{1}\right)}{32 M_{1}{ }^{6} d_{c}{ }^{2}}+\frac{105 M_{5}\left(\Xi_{1}-\Lambda_{1}\right)}{256 d_{c}{ }^{2} M_{1}{ }^{4}}-\frac{63 M_{2}{ }^{4}\left(\Xi_{1}-\Lambda_{1}\right)}{64 M_{1}{ }^{7} d_{c}{ }^{2}} \\
& +\frac{35 \Xi_{5}}{128 M_{1}{ }^{3} d_{c}{ }^{2}}-\frac{145 M_{3}{ }^{2}\left(\Xi_{1}-\Lambda_{1}\right)}{256 M_{1}{ }^{5} d_{c}{ }^{2}}+\frac{21 M_{2}{ }^{2} \Xi_{3}}{64 M_{1}{ }^{5} d_{c}{ }^{2}}-\frac{35 M_{3} \Xi_{3}}{128 d_{c}{ }^{2} M_{1}{ }^{4}},
\end{aligned}
$$




$$
\begin{aligned}
& -\frac{77 M_{4} M_{2}\left(\Xi_{1}-\Lambda_{1}\right)}{64 M_{1}{ }^{5} d_{c}{ }^{2}} \\
B_{2}^{(3)}= & -\frac{599 M_{3} M_{2}\left(\Xi_{1}-\Lambda_{1}\right)}{128 M_{1}{ }^{5} d_{c}{ }^{2}}+\frac{105 M_{2}{ }^{3}\left(\Xi_{1}-\Lambda_{1}\right)}{32 M_{1}{ }^{6} d_{c}{ }^{2}}-\frac{5 M_{3} \Xi_{2}}{16 d_{c}{ }^{2} M_{1}{ }^{4}} \\
& +\frac{385 M_{4}\left(\Xi_{1}-\Lambda_{1}\right)}{256 d_{c}{ }^{2} M_{1}{ }^{4}}+\frac{21 M_{2}{ }^{2} \Xi_{2}}{64 M_{1}{ }^{5} d_{c}{ }^{2}}+\frac{7 M_{2} \Xi_{3}}{128 d_{c}{ }^{2} M_{1}{ }^{4}}, \\
B_{2}^{(4)}= & -\frac{357 M_{2}{ }^{2}\left(\Xi_{1}-\Lambda_{1}\right)}{64 M_{1}{ }^{5} d_{c}{ }^{2}}+\frac{875 M_{3}\left(\Xi_{1}-\Lambda_{1}\right)}{256 d_{c}{ }^{2} M_{1}{ }^{4}}-\frac{35 \Xi_{3}}{128 M_{1}^{3} d_{c}{ }^{2}}-\frac{63 M_{2} \Xi_{2}}{128 d_{c}{ }^{2} M_{1}^{4}}, \\
B_{2}^{(5)}= & \frac{105 \Xi_{2}}{128 M_{1}{ }^{3} d_{c}{ }^{2}}+\frac{1617 M_{2}\left(\Xi_{1}-\Lambda_{1}\right)}{256 d_{c}{ }^{2} M_{1}{ }^{4}}, \quad B_{2}^{(6)}=-\frac{1155\left(\Xi_{1}-\Lambda_{1}\right)}{256 M_{1}{ }^{3} d_{c}{ }^{2}} .
\end{aligned}
$$

Note that the terms listed above are only potentially relevant, depending on which multi-critical model one wishes to consider. For an $m^{\prime}$ th multi-critical model all terms containing $M_{k}, k>m$, or $\Xi_{l}, l>m+1$, vanish in the double-scaling limit. We remind the reader that we assumed to have a non-symmetric potential and that the critical behaviour was associated with the endpoint $x$. In the case where the critical behaviour is associated with the endpoint $y$ all formulas in this section still hold provided $d_{c}$ is replaced by $-d_{c}, M_{k}$ by $J_{k}, \Xi_{k}$ by $\Lambda_{k}$ and $x$ by $y$.

\subsection{The Iteration for $F_{g}, \kappa_{g}$ and $\widehat{u}_{g}(p)$}

Having computed $u_{g}(p)$ and $v_{g}(p)$ (up to the zero mode) we may now proceed to calculate the free energy $F_{g}$ and the zero mode coefficient $\kappa_{g}$. This is done by rewriting $u_{g}$ and $v_{g}$ as total derivatives in the superloop insertion operators $\delta / \delta V(p)$ and $\delta / \delta \Psi(p)$ respectively, yielding the bosonic and doubly fermionic parts of $F_{g}$ as well as $\kappa_{g}$. The procedure to compute the bosonic part of the free energy $F_{g}^{\text {bos }}$ works just as in the hermitian matrix model described in ref. [四]. We have $F_{g}^{\text {bos }}=2 F_{g}^{\text {herm }}$.

From eq. (3.27) we see that $F_{g}$ scales as

$$
F_{g}=F_{g}^{\mathrm{bos}}+F_{g}^{\mathrm{ferm}} \sim a^{(2-2 g)(m+1 / 2)},
$$

just as the hermitian matrix model at its $m$ 'th multicritical point.

To obtain the doubly femionic part $F_{g}^{\text {ferm }}$ simply rewrite the basis functions $\chi^{(n)}(p)$ of $v_{g}(p)$ appearing in (3.26) in terms of the functions $\phi_{x}^{(n)}(p)$ which by eqs. (3.21) and (3.22) are nothing but total derivatives in $\delta / \delta \Psi(p)$. Doing this for $v_{g}(p)$ allows one to directly deduce the form of $F_{g}^{\text {ferm }}$ and $\kappa_{g}$. 囵

The explicit results for genus one are

$$
F_{1}^{\text {ferm }}=\left(\Xi_{1}-\Lambda_{1}\right)\left(-\frac{5 \Xi_{4}}{16 d_{c}{ }^{2} M_{1}{ }^{2}}+\frac{3 M_{2} \Xi_{3}}{8 d_{c}{ }^{2} M_{1}{ }^{3}}+\frac{5 M_{3} \Xi_{2}}{16 d_{c}{ }^{2} M_{1}{ }^{3}}-\frac{3 M_{2}{ }^{2} \Xi_{2}}{8 d_{c}{ }^{2} M_{1}{ }^{4}}\right)+\frac{\Xi_{2} \Xi_{3}}{8 d_{c}{ }^{2} M_{1}{ }^{2}}
$$

\footnotetext{
${ }^{4}$ Actually the presentation here is somewhat misleading. In order to compute the $u_{g}(p)$ and $v_{g}(p)$ by iteration in the way outlined in the previous subsection one needs to know the full $v_{1}(p), \ldots, v_{g-1}(p)$ including the zero mode coefficients $\kappa_{1}, \ldots, \kappa_{g-1}$. In practice one hence computes all quantities at genus $g$, i.e. $u_{g}, v_{g}, \kappa_{g}$ and $F_{g}$, before proceeding to genus $(g+1)$.
} 
and

$$
\kappa_{1}=\frac{5 \Xi_{4}}{16 M_{1}^{2} d_{c}}-\frac{3 M_{2} \Xi_{3}}{8 M_{1}^{3} d_{c}}-\frac{5 M_{3} \Xi_{2}}{16 M_{1}^{3} d_{c}}+\frac{3 M_{2}^{2} \Xi_{2}}{8 M_{1}^{4} d_{c}} .
$$

This expression may of course be alternatively obtained by taking the double scaling limit of the genus one result of ref. [6] computed away from the scaling limit.

Before presenting explicit results for genus two, let us describe the general structure of $F_{g}^{\text {ferm }}$ for $g \geq 1$

$$
F_{g}^{\mathrm{ferm}}=\sum_{\alpha_{j}, \beta_{i}}\left\langle\alpha_{1}, \ldots, \alpha_{s}, \beta_{1}, \beta_{2} \mid \alpha\right\rangle_{g} \frac{\Xi_{\beta_{1}} \Xi_{\beta_{2}} M_{\alpha_{1}} \ldots M_{\alpha_{s}}}{M_{1}{ }^{\alpha}{d_{c}}^{g+1}},
$$

where we write $\Xi_{1}$ for $\left(\Xi_{1}-\Lambda_{1}\right)$ and where the brackets denote rational numbers as before. The $\alpha_{j}, \beta_{i}, \alpha$ and $s$ are subject to the constraints

$$
\alpha=2 g+s, \quad \text { and } \quad \sum_{j=1}^{s}\left(\alpha_{j}-1\right)=3 g+2-\beta_{1}-\beta_{2},
$$

where $\alpha_{i} \in[2,3 g]$ and $k_{i} \in[1,3 g+1]$. Similar relations hold for $F_{g}^{\text {bos }}$ 幽.

The result for genus two reads

$$
\begin{aligned}
F_{2}^{\mathrm{ferm}}= & \left(\Xi_{1}-\Lambda_{1}\right)\left[\frac{1015 \Xi_{5} M_{3}}{128 M_{1}{ }^{5} d_{c}{ }^{3}}-\frac{375 \Xi_{4} M_{2} M_{3}}{16 M_{1}{ }^{6} d_{c}{ }^{3}}\right. \\
& +\frac{315 \Xi_{4} M_{2}{ }^{3}}{16 M_{1}{ }^{7} d_{c}{ }^{3}}+\frac{385 \Xi_{4} M_{4}}{64 M_{1}{ }^{5} d_{c}{ }^{3}}+\frac{1323 \Xi_{2} M_{2}{ }^{5}}{64 M_{1}{ }^{9} d_{c}{ }^{3}}+\frac{693 \Xi_{6} M_{2}}{64 M_{1}{ }^{5} d_{c}{ }^{3}} \\
& -\frac{1155 \Xi_{7}}{256 M_{1}{ }^{4} d_{c}{ }^{3}}-\frac{63 \Xi_{2} M_{2} M_{5}}{4 M_{1}{ }^{6} d_{c}{ }^{3}}-\frac{525 \Xi_{5} M_{2}{ }^{2}}{32 M_{1}{ }^{6} d_{c}{ }^{3}}-\frac{1155 \Xi_{3} M_{2} M_{4}}{64 M_{1}{ }^{6} d_{c}{ }^{3}} \\
& +\frac{315 \Xi_{3} M_{5}}{64 M_{1}{ }^{5} d_{c}{ }^{3}}-\frac{2175 \Xi_{3} M_{3}{ }^{2}}{256 M_{1}{ }^{6} d_{c}{ }^{3}}+\frac{675 \Xi_{3} M_{2}{ }^{2} M_{3}}{16 M_{1}{ }^{7} d_{c}{ }^{3}}-\frac{1785 \Xi_{2} M_{3} M_{4}}{128 M_{1}{ }^{6} d_{c}{ }^{3}} \\
& -\frac{495 \Xi_{2} M_{2}{ }^{3} M_{3}}{8 M_{1}{ }^{3} d_{c}{ }^{3}}+\frac{2205 \Xi_{2} M_{2}{ }^{2} M_{4}}{64 M_{1}{ }^{7} d_{c}{ }^{3}}-\frac{1323 \Xi_{3} M_{2}{ }^{4}}{64 M_{1}{ }^{8} d_{c}{ }^{3}}+\frac{8175 \Xi_{2} M_{3}{ }^{2} M_{2}}{256 M_{1}{ }^{7} d_{c}{ }^{3}} \\
& \left.+\frac{1155 \Xi_{2} M_{6}}{256 M_{1}{ }^{5} d_{c}{ }^{3}}\right]-\frac{21 \Xi_{2} \Xi_{5} M_{2}}{16 M_{1}{ }^{5} d_{c}{ }^{3}}+\frac{105 \Xi_{2} \Xi_{6}}{128 M_{1}^{4} d_{c}{ }^{3}}-\frac{145 \Xi_{2} \Xi_{4} M_{3}}{128 M_{1}{ }^{5} d_{c}{ }^{3}} \\
& +\frac{105 \Xi_{2} \Xi_{4} M_{2}{ }^{2}}{64 M_{1}{ }^{6} d_{c}{ }^{3}}+\frac{21 \Xi_{3} \Xi_{4} M_{2}}{64 M_{1}{ }^{5} d_{c}{ }^{3}}-\frac{35 \Xi_{3} \Xi_{5}}{128 M_{1}{ }^{4} d_{c}{ }^{3}}-\frac{63 \Xi_{2} \Xi_{3} M_{2}{ }^{3}}{32 M_{1}{ }^{7} d_{c}{ }^{3}} \\
& -\frac{35 \Xi_{2} \Xi_{3} M_{4}}{32 M_{1}{ }^{5} d_{c}{ }^{3}}+\frac{195 \Xi_{2} \Xi_{3} M_{2} M_{3}}{64 M_{1}{ }^{6} d_{c}{ }^{3}} .
\end{aligned}
$$

Of course so far we have determined only the coefficients $F_{g}$ of the genus expansion of the free energy (cf. eq. (2.7)). For an $m$ 'th multicritical model the relevant expansion parameter in the double-scaling limit is the string coupling constant $\alpha=a^{-2 m-1} N^{-2}$. If we introduce the bosonic and fermionic scaling moments $\mu_{k}$ and $\tau_{k}$ by (cf. eqs. (3.6) and (3.12)) 


$$
\begin{gathered}
M_{k}=a^{m-k} \mu_{k}, \quad k \in[1, m], \\
\left(\Xi_{1}-\Lambda_{1}\right)=a^{m+1 / 2} \tau_{1}, \quad \Xi_{l}=a^{m-l+3 / 2} \tau_{l}, \quad l \in[2, m+1],
\end{gathered}
$$

we get by replacing $M_{k}$ by $\mu_{k}$ and setting $M_{k}$ equal to zero for $k>m$ as well as replacing $\Xi_{l}$ by $\tau_{l}$ and setting $\Xi_{l}$ to zero for $l>m+1$ in the formulas above exactly the coefficients of the expansion in the string coupling constant. Needless to say that these results apply for non-symmetric potentials where the critical behaviour is associated with the endpoint $y$ as well by performing the usual replacements.

For the zero mode coefficient at genus two we find

$$
\begin{aligned}
& \kappa_{2}=\frac{495 \Xi_{2} M_{3} M_{2}^{3}}{8 d_{c}{ }^{2} M_{1}{ }^{8}}+\frac{63 \Xi_{2} M_{5} M_{2}}{4 d_{c}{ }^{2} M_{1}{ }^{6}}-\frac{315 \Xi_{3} M_{5}}{64 d_{c}{ }^{2} M_{1}{ }^{5}}+\frac{1323 \Xi_{3} M_{2}{ }^{4}}{64 d_{c}{ }^{2} M_{1}{ }^{8}} \\
& -\frac{675 \Xi_{3} M_{3} M_{2}{ }^{2}}{16 d_{c}^{2} M_{1}{ }^{7}}+\frac{2175 \Xi_{3} M_{3}{ }^{2}}{256 d_{c}{ }^{2} M_{1}{ }^{6}}-\frac{8175 \Xi_{2} M_{3}{ }^{2} M_{2}}{256 d_{c}{ }^{2} M_{1}{ }^{7}}-\frac{385 \Xi_{4} M_{4}}{64 d_{c}{ }^{2} M_{1}{ }^{5}} \\
& -\frac{2205 \Xi_{2} M_{4} M_{2}{ }^{2}}{64 d_{c}{ }^{2} M_{1}{ }^{7}}+\frac{375 \Xi_{4} M_{3} M_{2}}{16 d_{c}{ }^{2} M_{1}{ }^{6}}-\frac{1155 \Xi_{2} M_{6}}{256 d_{c}{ }^{2} M_{1}{ }^{5}}+\frac{1785 \Xi_{2} M_{4} M_{3}}{128 d_{c}{ }^{2} M_{1}{ }^{6}} \\
& -\frac{693 \Xi_{6} M_{2}}{64 d_{c}^{2} M_{1}{ }^{5}}-\frac{315 \Xi_{4} M_{2}{ }^{3}}{16 d_{c}{ }^{2} M_{1}{ }^{7}}+\frac{525 \Xi_{5} M_{2}{ }^{2}}{32 d_{c}{ }^{2} M_{1}{ }^{6}}-\frac{1015 \Xi_{5} M_{3}}{128 d_{c}{ }^{2} M_{1}{ }^{5}} \\
& -\frac{1323 \Xi_{2} M_{2}^{5}}{64 d_{c}^{2} M_{1}^{9}}+\frac{1155 \Xi_{3} M_{4} M_{2}}{64 d_{c}^{2} M_{1}^{6}}+\frac{1155 \Xi_{7}}{256 d_{c}^{2} M_{1}^{4}} \text {. }
\end{aligned}
$$

We obtained these results with the aid of a Maple program which performs the iteration up to arbitrary genus. [ In practice the expressions become quite lengthy, e.g. $F_{3}^{\text {ferm }}$ consists of 114 terms.

For the knowledge of the full $W_{g}(\mid p)$ it remains to compute $\widehat{u}_{g}(p)$. This is of course done by applying $\delta / \delta V(p)$ to $F_{g}^{\text {ferm }}$. The general structure of $\widehat{u}_{g}(p)$ turns out to be [6]

$$
\widehat{u}_{g}(p)=\sum_{k=1}^{3 g+1} \widehat{A}_{g}^{(k)} \chi^{(k)}(p),
$$

where

$$
\widehat{A}_{g}^{(k)}=\sum_{\alpha_{j}, \beta_{1}, \beta_{2}}\left\langle\alpha_{1}, \ldots, \alpha_{s}, \beta_{1}, \beta_{2} \mid \alpha\right\rangle_{g, k} \frac{M_{\alpha_{1}} \ldots M_{\alpha_{s}} \Xi_{\beta_{1}} \Xi_{\beta_{2}}}{M_{1}{ }^{\alpha} d_{c}{ }^{g+1}}
$$

underlying the conditions

$$
\alpha=s+3 g, \quad \text { and } \quad \sum_{j=1}^{s}\left(\alpha_{j}-1\right)=4+3 g-k-\beta_{1}-\beta_{2},
$$

with $\alpha_{j} \in[2,3 g+1]$ and $\beta_{i} \in[1,3 g+2]$. Due to space let us only state the genus one results

\footnotetext{
${ }^{5}$ The reader interested in higher-genus results should feel free to contact the author.
} 


$$
\begin{aligned}
\widehat{A}_{1}^{(1)}= & \frac{5 \Xi_{2} \Xi_{4}}{32 d_{c}{ }^{2} M_{1}{ }^{2}}+\frac{3 M_{2} \Xi_{2} \Xi_{3}}{16 d_{c}{ }^{2} M_{1}{ }^{3}}-\frac{35\left(\Xi_{1}-\Lambda_{1}\right) \Xi_{5}}{32 d_{c}{ }^{2} M_{1}{ }^{2}} \\
& +\frac{15 M_{3}\left(\Xi_{1}-\Lambda_{1}\right) \Xi_{3}}{32 d_{c}{ }^{2} M_{1}{ }^{3}}+\frac{15 M_{2}\left(\Xi_{1}-\Lambda_{1}\right) \Xi_{4}}{16 d_{c}{ }^{2} M_{1}{ }^{3}}-\frac{9 M_{2}{ }^{2}\left(\Xi_{1}-\Lambda_{1}\right) \Xi_{3}}{16 d_{c}{ }^{2} M_{1}{ }^{4}}, \\
\widehat{A}_{1}^{(2)}= & -\frac{15\left(\Xi_{1}-\Lambda_{1}\right) \Xi_{4}}{16 d_{c}{ }^{2} M_{1}{ }^{2}}+\frac{3 \Xi_{2} \Xi_{3}}{8 d_{c}{ }^{2} M_{1}{ }^{2}}+\frac{3 M_{2}\left(\Xi_{1}-\Lambda_{1}\right) \Xi_{3}}{4 d_{c}{ }^{2} M_{1}{ }^{3}} \\
& +\frac{5 M_{3}\left(\Xi_{1}-\Lambda_{1}\right) \Xi_{2}}{16 d_{c}{ }^{2} M_{1}{ }^{3}}-\frac{3 M_{2}{ }^{2}\left(\Xi_{1}-\Lambda_{1}\right) \Xi_{2}}{8 d_{c}{ }^{2} M_{1}{ }^{4}}, \\
\widehat{A}_{1}^{(3)}= & -\frac{15\left(\Xi_{1}-\Lambda_{1}\right) \Xi_{3}}{16 d_{c}{ }^{2} M_{1}{ }^{2}}+\frac{25 M_{2}\left(\Xi_{1}-\Lambda_{1}\right) \Xi_{2}}{32 d_{c}{ }^{2} M_{1}{ }^{3}}, \quad \widehat{A}_{1}^{(4)}=-\frac{35\left(\Xi_{1}-\Lambda_{1}\right) \Xi_{2}}{32 d_{c}{ }^{2} M_{1}{ }^{2}} .
\end{aligned}
$$

This concludes our analysis of the double-scaling limit for generic potentials.

The case of symmetric bosonic and generic fermionic potentials was considered in ref. [四] where a doubling of degrees of freedom was observed for genus zero. With the methods presented in this paper one can see that this holds for higher genera as well, i.e. the free energy here takes the form

$$
F_{g}^{\mathrm{symm}}=2 F_{g}^{\mathrm{bos}}+F_{g}^{\mathrm{ferm}, \mathrm{x}}+F_{g}^{\mathrm{ferm}, \mathrm{y}},
$$

where $F_{g}^{\text {bos }}$ denotes the bosonic part of the free energy for generic potentials, $F_{g}^{\text {ferm,x }}$ and $F_{g}^{\text {ferm,y }}$ denote the doubly fermionic parts in the generic case where the critical behaviour is associated with the endpoints $x$ and $y=-x$ respectively (cf. eq. (3.35)). If one chooses to take the fermionic potential to be symmetric as well the doubly fermionic part of the free energy will vanish, which may be directly deduced from the results of ref. [7].

\section{Conclusions}

We have studied the double scaled supereigenvalue model in the moment description. The $m$ 'th multi-critical point was identified and the scaling properties of the moments and basis functions derived. The iterative scheme for the calculation of higher-genus contributions could be optimized to produce only terms relevant in the double-scaling limit. The general form of the free energy and the one-superloop correlators at genus $g$ were found. We presented explicit results up to genus two.

We believe that this paper shows once more the effectiveness of the iterative scheme and the moment description of Ambjørn et al. The analogy of structures in the hermitian one matrix model and in the supereigenvalue model continues to hold. It is interesting to note that the analogous computations for the hermitian

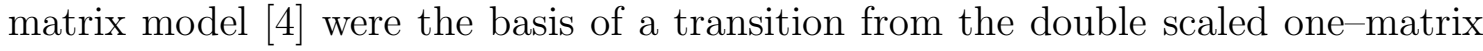
to the Kontsevich model [12]. One might speculate that our results are connected to properties of the moduli space of super-Riemann surfaces. 
There are more interesting unanswered questions to be adressed, such as the supersymmetric generalization of two and multi-matrix models, where one would hope to reach new minimal superconformal models, or the generalization of matrix models in external fields, perhaps a step towards a matrix based formulation of the supereigenvalue model.

Acknowledgements: I am particularly grateful to E. Abdalla and A. Zadra for pointing out the situation at the $m=1$ fixed-point to me. Moreover I wish to thank P. Adamietz, G. Akemann, J. Bischoff and O. Lechtenfeld for interesting and valuable discussions. 


\section{References}

[1] L. Alvarez-Gaumé, H. Itoyama, J. L. Mañes and A. Zadra, Int. J. Mod. Phys. A7 (1992) 5337.

[2] E. Abdalla, M. C. B. Abdalla, D. Dalmazi and A. Zadra, 2D-Gravity in NonCritical Strings, Lecture Notes in Physics m20 (Springer, Berlin, 1994).

[3] M. Takama, Phys. Lett. B 284 (1992) 248.

[4] J. Ambjørn, L. Chekhov, C. F. Kristjansen and Yu. Makeenko, Nucl. Phys. B404 (1993) 127.

[5] L. Alvarez-Gaumé, K. Becker, M. Becker, R. Emparan and J. Mañes, Int. J. Mod. Phys. A8 (1993) 2297.

[6] J.C. Plefka, preprint DESY-95-015, ITP-UH-04-95, hep-th/9501120, to appear in Nucl. Phys. B.

[7] K. Becker and M. Becker, Mod. Phys. Lett. A8 (1993) 1205.

[8] I. N. McArthur, Mod. Phys. Lett. A8 (1993) 3355.

[9] J. M. Figueroa-O'Farrill and S. Stanciu, Phys. Lett. B 316 (1993) 282.

I. N. McArthur, J. Phys. A 27 (1994) 4161.

[10] A. Zadra and E. Abdalla, Nucl. Phys. B432 (1994) 163.

[11] V. A. Kazakov, Mod. Phys. Lett. A4 (1989) 2125.

[12] J. Ambjørn and C. F. Kristjansen, Mod. Phys. Lett. A8 (1993) 2875. 